\title{
Communication-constrained $p$-Center Problem for Event Coverage in Theme Parks
}

\author{
Gürkan Solmaz ${ }^{1}$, Kemal Akkaya ${ }^{2}$ and Damla Turgut ${ }^{1}$ \\ ${ }^{1}$ Department of Elect. Engineering and Computer Science, University of Central Florida, Orlando, FL 32816 \\ ${ }^{2}$ Department of Computer Science, Southern Illinois University, Carbondale, IL 62901
}

\begin{abstract}
Wireless sensor networks with mobile sinks can be deployed for efficient handling of the events that may occur in a theme park. In such a case, the success of event handling depends on the positions of the mobile sinks and the selection of the most suitable sink to cover an event. While this problem can be solved by using the classical vertex $p$-center problem, such a solution does not guarantee connectivity among the mobile sinks. The connectivity among mobile sinks is crucial since they need to communicate to share information and perform collaborative event handling.

In this paper, we introduce a new variant of vertex $p$ center problem which we name communication-constrained $p$ center problem. We propose an exact algorithm as a solution based on identifying connected subnets among the vertices. The performance of the proposed solution is validated through simulations with respect to other approaches as well as the unconstrained case.
\end{abstract}

\section{INTRODUCTION}

Theme parks are large entertainment areas which are visited by many people every day and have important economic impact on tourism industry. The areas of the theme parks consist of attractions in which the visitors spend most of their time. There are roads connecting the attractions to each other and to the exit gates. The attractions are the most crowded regions and have security vulnerabilities causing incidents such as violence or robbery. Furthermore, as in any other crowded environments, emergency situations may occur in the theme park. Whether it be an incident or emergency, we refer to these cases simply as events. Operators of the theme parks should handle these events quickly and efficiently to maintain the safety of the theme park.

Considering fundamental characteristics of theme parks such as being large bordered areas and limited use of transportation vehicles, this problem relates to full coverage of the events and can be addressed by deploying a wireless sensor network (WSN) with mobile sinks. Specifically, sensors in different parts of regions of attractions collect information regarding the events and relay this information to a number of mobile sinks. These sinks are electric safety vehicles which are controlled by humans. In addition to WSNs, crowdsensing via smart phones can also be applied to collect such information. The problem then reduces to the effective placement of mobile sinks to locations such that each event can be covered in the minimal amount of time by sending the closest mobile sink to the location of the event.

This problem can be modeled as a vertex $p$-center problem [1] which aims to minimize the maximum travel distance for each sink. Specifically, we represent the theme park with a weighted graph. In this graph, the edges and the vertices have dynamic values that change according to the current movement and the positions of the visitors. The edges correspond to the roads and the weights of the edges are the travel times of mobile sinks on these roads. The vertices correspond to the attractions and the weights of the vertices are the event probabilities of the attractions. While the solution of vertex $p$-center problem produces optimal places of the mobile sinks at the attractions, the sinks should still be able to communicate with each other to share information and request help from others if needed. In this case, there should be a connected network among the mobile sinks. This brings additional constraint to the problem which cannot be handled with the use of vertex $p$-center problem.

In this paper, we introduce a new variant of vertex $p$-center problem which we call communication-constrained $p$-center problem. In this new variant, the connected sinks form wireless network which consists of a subset of the vertices. The possible locations of the mobile sinks is modeled with connectivity graph. We also propose an exact algorithm to solve this new problem. Our proposed algorithms has two steps: finding the connected subgraphs and placing mobile sinks to the vertices on these subgraphs such that the maximum distance from attractions to mobile sinks is minimized. The positions of the mobile sinks are updated in discrete time intervals to place the sinks either on or close to the attractions which have higher event probabilities. The proposed approach of coverage with WSNs with mobile sinks can be applicable to other scenarios such as security of airports and shopping malls.

We implemented and tested our approach under a variety of conditions. Compared to baselines, the proposed approaches provide reduced event handling times and increase the chance of handling events on time. In addition, the results revealed that connectivity constraint increases the event handling times, while it gives independence to the network by eliminating the need of a global monitoring infrastructure.

The rest of the paper is organized as follows. We briefly discuss the related work in Section II. In Section III, we give background on the $p$-center problem and describe the theme park model. In Section IV, we propose the new problem and the exact algorithm for its solution. The simulation study is included in Section V and we finally conclude in Section VI.

\section{RELATED WORK}

In order to manage the mobility and the positioning of the mobile sink nodes, there are various strategies proposed in the literature. Younis and Akkaya [2] survey the techniques for node placement strategies used in WSNs for effective 
optimization. Among all these techniques, we focus on $p$ center problems that suit our needs in a theme park.

The $p$-center is an old problem which is NP-hard in general graphs [1]. Different versions of $p$-center problem have been studied in the past to propose heuristics to solve it. Since we propose an exact algorithm, we only summarize the literature dealing with the exact solutions. For instance, Kariv and Hakimi ([1],[3]) are the first to define the absolute and vertex $p$-center and $p$-median network location problems and propose exact algorithms for solving these problems. Özsoy and Pinar [4] propose an exact algorithm to solve a different version, called the capacitated vertex $p$-center problem. In this case, each client is labeled with a demand quantity and assignment of the $p$-centers is constrained. The total demand by the clients cannot exceed the capacity of the facility. A good summary of the literature for the solutions of both the capacitated and the uncapacitated $p$-median problems can be found in Reese [5].

The connected $p$-center problem studied by Yen [6] was the closest to our problem. In this case, the $p$ vertices should be connected on the same graph. The problem is solved with exact algorithms on trees [7], block graphs [6] and trees [8] with forbidden vertices. Our problem is different in the sense that the connectivity should be through wireless communication not physical paths.

There are other applications of $p$-center problem in WSNs mostly in the context of clustering [9][10]. In these works, the idea is to minimize the packet delay from each sensor to the sink node but there is no restriction for the connectivity of the sink nodes. Our problem is different from them in terms of its communication constraint.

\section{PRELIMINARIES}

\section{A. Background on p-center problem}

The $p$-center problem consists of $p$ facilities and clients (i.e., vertices). Each client is assigned to a facility. The problem is to place the $p$ facilities on the network in a way that the maximum distance between a client and the facility assigned to it is minimized. There are two major variants of the $p$-center problem: absolute and vertex $p$-center. In the absolute $p$-center problem, the facilities can be located anywhere in the network, including vertices and any points on the edges. In the vertex $p$ center, the facilities can be located on top of the vertices only. There is another variant called the connected vertex $p$-center $(\mathrm{CpC})$ problem. In this case, the selected $p$ facilities should be placed on top of the vertices and the vertices should be connected to each other via physical paths.

There are also other variants of the problem if different graph models are considered. For instance, if the vertices are weighted, then the problem is called vertex-weighted $p$-center; otherwise, it is called vertex-unweighted $p$-center problem. In case of the vertex-weighted $p$-center problem, the distance values are multiplied by the weight values of the vertices. The weights of the vertices are considered as the demands of the clients.

Finally, depending on the goal the $p$-median problem is defined that is finding the locations of $p$ facilities on the network such that the sum of the shortest distances between the facilities and the clients is minimum. It has also two variants, the absolute and the vertex $p$-median problem as in the $p$-center problem. If the vertices are weighted, it is called the vertex-weighted $p$-median problem and the vertexunweighted $p$-median problem in the other case. Considering the connectivity constraint, the vertices of the $p$ facilities must be connected and in this case it is called the connected vertex $p$-median problem.

\section{B. Wireless sensor network model}

We describe the model of a WSN with mobile sinks specifically designed to allow the operator of the theme park to efficiently and promptly handle events. The network consists of two types of nodes.

Static sensor nodes are deployed throughout the theme park. Their capabilities are limited to sensing events occurring in their physical vicinity, and the transmission of their observations to the mobile sinks using hop-by-hop transmissions. The sensor nodes are passive observers, they cannot take actions to handle events and may stay idle when there is no event or they may sense environmental data for regular monitoring purposes. We assume that static nodes are deployed inside the attractions of the theme park. In this study, we focus on mobile sink positioning approach for event coverage. For a complete model of the system, an appropriate routing protocol for sensor nodes must be chosen [11].

Mobile sinks exist in limited numbers. They patrol inside the attractions with the goal of data collection and the event coverage. The mobile sinks have the ability to move fast and share information among each other. In a real scenario, we may consider the mobile sinks as the electronic transportation vehicles used by the security personnel with a wireless communication device. If there is an event happening in an attraction, one of the sinks is responsible for moving to the region and handling the event. Otherwise, the sinks do their patrolling duty in their allocated attractions.

\section{Theme park modeling}

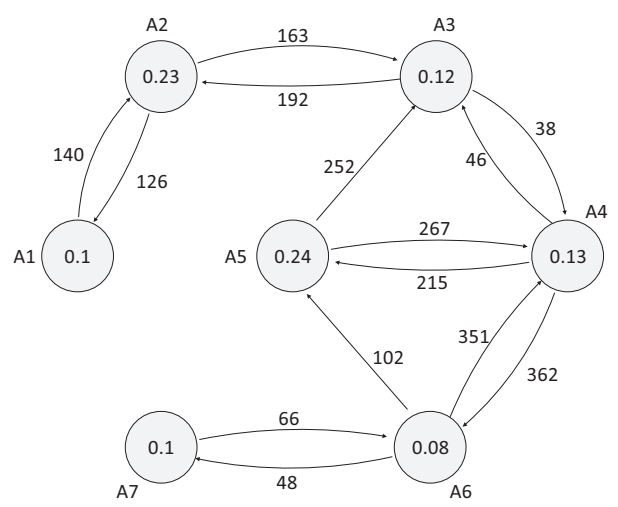

Fig. 1. The theme park graph model.

Considering the attractions and the roads in a park, we model the theme park with a graph model. The vertices of the graph represent the attractions while the edges represent the roads that connect the attractions. Note that not every pair of vertices have edges between them, but every vertex has a connection to at least one other vertex and therefore the 


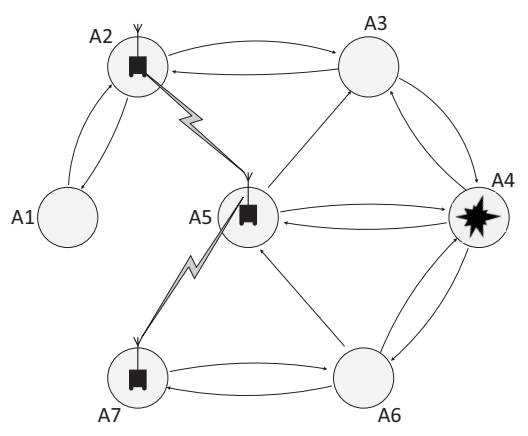

Fig. 2. The connectivity of the mobile sinks in the attractions and an event.

graph is connected. Fig. 1 illustrates the theme park graph model. In this figure, $A 1, A 2, \ldots, A 7$ represent the attractions. The possibility of incidents/events at attractions are shown as the weight values which are displayed on top of the vertices. The time estimates for the mobile sinks to travel between the attractions are shown as the weights of the edges.

\section{Event Coverage With \\ Communication-Constrained $p$-Center Problem}

\section{A. Motivation}

The problem of mobile sink placement can be solved by using one of the existing heuristics of the vertex $p$-center problem [5]. However, we have an additional constraint that needs to be satisfied. The mobile sinks should be directly connected to each other in order to share information of the events and take collaborative actions if needed. Therefore, they should always preserve a connected topology as illustrated in Fig. 2. In this figure, the wireless connections between the mobile sinks and an event in $A 4$ are illustrated whereas the arrows represent the roads between the attractions.

Because of this additional constraint, we face with a new variant of the vertex $p$-center problem in which the facilities (i.e., sinks) need to communicate with each other. We call it communication-constrained p-center problem.

In this problem, a facility is considered connected whenever it is in the transmission range of at least one other facility while all facilities form a connected graph. As aforementioned, the original connected $p$-center problem forces the connectivity of the facilities through the physical paths which is different than our problem. In the new problem, even if the facilities are connected via physical paths (roads), there may not be a wireless communication among them due to limited radio range. Therefore, the solution should use two distinct graphs. The distances from facilities to clients must be computed using the theme park graph while the connectivity between the facilities must be checked with the connectivity graph.

\section{B. Problem formulation}

The formulation of the communication-constrained $p$-center problem is as follows. Given a connected graph $G$, let $V=$ $\left\{v_{1}, v_{2}, \ldots, v_{n}\right\}$ be the set of its vertices. We assume that each vertex $v_{i}$ has a weight value $w\left(v_{i}\right)$ and $w\left(v_{i}\right)>0, \forall v_{i} \in V$ which corresponds to the demand of the vertex. $d\left(v_{i}, v_{j}\right)$ is the length of the shortest path from a vertex $v_{i}$ to a vertex $v_{j} . G^{c}$ is the connectivity graph which is explained in section
IV-C. There are $p$ facilities that can be placed only on top of the vertices. The facilities should be placed on optimal $p$ vertices, such that the maximum distance between the facilities and the vertices is minimized. Moreover, $p$ facilities must be connected, in a way that each facility must be located within the communication range $R$ of at least one other facility.

The objective is to find the subset of vertices $F=\left\{f_{1}, f_{2}, \ldots, f_{p}\right\}$, where $F \subset V$ and $|F|=p$ to locate the facilities with the following goal:

$$
\text { Min } \eta(F) \quad \text { s.t. } \quad F \text { is connected on } G^{c}
$$

where

$$
\eta(F)=\max _{1 \leq i \leq n}\left\{\min _{1 \leq j \leq p}\left\{\left(w\left(v_{i}\right) \cdot d\left(f_{j}, v_{i}\right)\right\}\right\} .\right.
$$

The minimum value of $\eta(F)$ gives the optimal subset of vertices $F$ for facility locations. For each client (vertex) $v_{i}$ the minimum distance is calculated by the shortest path distance $d\left(f_{j}, v_{i}\right)$ between a facility in $f_{j}$ and a client (vertex) $v_{i}$ multiplied by the weight of the client $w\left(v_{i}\right)$.

This problem is challenging in the sense that the $p$-center problem is NP-hard [1] by reduction to the dominating set problem. Considering the definition of the communicationconstrained $p$-center problem, one can easily show that the problem is NP-hard on general graphs. Assuming the case that there exist communication paths between all pairs of vertices in graph $G$, the constraint disappears due to the fact that one can place facilities in any $p$ locations and any selected $p$ vertices allow the facilities to form communication paths among them. The problem of finding $p$ vertices (finding the subset $F$ ) becomes exactly the same as the original $p$-center problem.

\section{Proposed approach}

According to the Euclidean distances between the vertices, we consider a graph $G^{c}$, called the connectivity graph. The edges of the connectivity graph are created among each pair of vertices if the Euclidean distance among them is less than or equal to $R$.

Considering the moderate sizes of the problem in real-life context in the theme parks, we propose an exact algorithm for solving the communication-constrained $p$-center problem. This algorithm has two steps. In the first step, we compute the connected subgraphs with $p$ vertices using the connectivity graph $G^{c}$. In the second step, the facilities will be placed to the vertices of these connected subgraphs using the $p$-center solution to minimize the maximum or total distance.

For the first step, the set of connected subgraphs $S\left[G^{c}\right]=$ $\left\{S_{1}, S_{2}, \ldots, S_{k}\right\}$ is found which includes the candidates for the best locations of $p$ facilities where $k$ is the number of candidate subgraphs with $\left|S_{i}\right|=p, \forall S_{i} \in S\left[G^{c}\right]$.

For timely event coverage, we apply $p$-center problem to the connected subgraphs with $p$ vertices using $G$ and and compute the new positions of the mobile sinks. Depending on the goal, we can choose a different metric. For instance, if we would like to minimize the maximum distance from an attraction to the closest sink, this placement approach is referred to as $p$-center positioning $(\mathrm{PcP})$. If the total distance is to be minimized, then this approach is called p-median positioning $(\mathrm{PmP}) . \mathrm{We}$ implemented these two strategies in this paper. 
Let the locations of vertices stored in a set $F$. The subset $F$ with $p$ vertices that minimizes the maximum (or total) weighted shortest path distance from the facilities to the clients is guaranteed to be one of the candidates since these candidates are subsets of $G^{c}$ that comply with the connectivity requirement, $F \in S\left[G^{c}\right]$. The weighted distances from the vertices of graph $G$ (clients) to the candidates (facilities) are computed to find the best candidate $F$ that minimizes the maximum (total) distance to the clients. The weighted distances between the connected subsets and vertices are calculated and shown as the matrix $W$, such that

$$
W_{S_{k}, v_{i}}=d\left(S_{k}, v_{i}\right) \cdot w\left(v_{i}\right)
$$

where $d\left(S_{k}, v_{i}\right)$ is the minimum shortest path distance from any vertex of $S_{k}$ to the vertex $v_{i}$. The pseudo-code for the algorithm is shown in Algorithm 1.

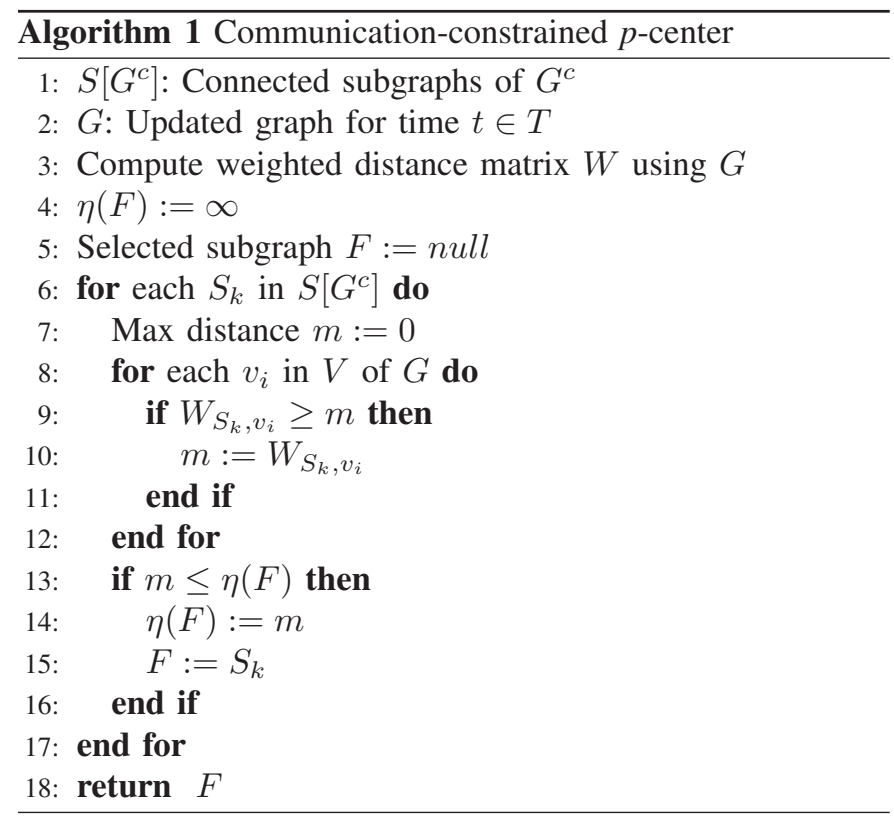

Since the weights of the edges and attractions change dynamically in the theme park, the mobile sinks have discrete location update times $t \in T$. At each update time $t$, one of the sinks is chosen as the master which runs Algorithm 1 using the new weights and assigns the new positions to the other sinks (i.e., slave). The assignment is done by sharing the new attraction allocation list with the sinks. Throughout the operation of the network, $G^{c}$ does not change since the attractions have static locations and therefore $G^{c}$ is initially provided to the master sink.

Fig. 3 illustrates the behaviors of the PcP and PmP approaches. In this figure, we consider one mobile sink and do not illustrate the roads between the attractions for simplicity. Let us assume that the positions of the attractions represent the scaled Euclidean distances between the attractions and the event probabilities of all the attractions are equal. According to PcP, the sink needs to be placed at the attraction which is located in the middle whereas in PmP, it is placed in a location closed to most of the attractions.

\section{Algorithm complexity}

The first step of finding all connected subgraphs in a graph takes $O(e n k)$ time [12], where $e, n$ and $k$ (i.e., $\left.\left|S\left[G^{c}\right]\right|\right)$ are the

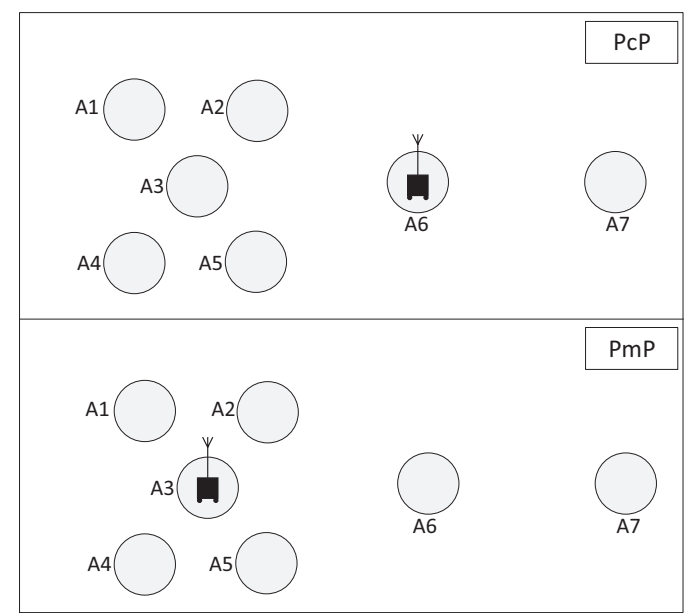

Fig. 3. Mobile sink positioning with PcP and PmP.

number of edges, the number of vertices of $G$, and the number of connected subgraphs respectively. This step is assumed to be done offline before the real-time operation of the sinks and the candidate subgraph list is provided to the master sink.

All weighted distances $W_{S_{k}, v_{i}}$ are computed initially, which takes $O\left(n^{3}\right)$ for general graphs. The algorithm then iterates $k$ times for each $S$ in $S\left[G^{c}\right]$ and $n$ times for each $v \in V$ of $G$. Hence, overall complexity of the algorithm is $O(k n)$. The efficiency of the algorithm mainly depends on $k$ since $1 \leq$ $k \leq C(n, p)$. In the best case, there exists only one connected subgraph with $p$ vertices $\left(O\left(n^{3}\right)\right)$ while in the worst-case the connectivity graph $G^{c}$ is a complete graph $(k=C(n, p))$.

\section{Simulation STUdy}

\section{A. Simulation setup}

To assess the performance of the proposed algorithm, we conducted simulations with the following settings. Initially, the attractions are generated at an empty terrain of a theme park with random event probabilities assigned as the weight values. The locations of the attractions are determined randomly and according to specified minimum and maximum Euclidean distances among attractions. The events are generated by a Poisson process. The event probabilities of all the attractions are updated according to the model described in [13], [14] which is based on the history of the events. We define a certain active time period for an event and once it expires, the event is assumed to end. The active times of the events are randomly generated. The weights of the edges are initially generated by using a lower bound and an upper bound considering the physical distances and the possible changes in mobile sink travel times due to pedestrian flows on the roads. These weights are changed by a ratio at discrete update times. The speed of the mobile sinks are set to their maximum (i.e., the speed when the road is empty). While the sinks are considered as same type of vehicles with a fixed maximum speed, the speed of each sink changes during the simulation because of the slowdowns caused by movements of pedestrians on the roads. Although the mobile sinks with higher speeds handle events faster, they share the roads with the pedestrians. Therefore, their maximum speed is limited for safety of the 
TABLE I

SIMULATION PARAMETERS

\begin{tabular}{|l|l|}
\hline simulation time $(T)$ & 10 hours \\
\hline terrain size & 500 x $500 \mathrm{~m}$ \\
\hline number of attractions $(n)$ & 15 \\
\hline min distance among attractions & $50 \mathrm{~m}$ \\
\hline max distance among attractions & $250 \mathrm{~m}$ \\
\hline node degree of $G$ & 4 \\
\hline sink update time $t$ & $30 \mathrm{~min}$ \\
\hline sink transmission range & $100 \mathrm{~m}$ \\
\hline event probability change rate & 0.01 \\
\hline expected number of events & 100 \\
\hline min active time of events & $200 \mathrm{sec}$ \\
\hline max active time of events & $600 \mathrm{sec}$ \\
\hline edge weight change rate & 0.20 \\
\hline max edge weight difference & $400 \%$ \\
\hline max mobile sink speed & $1.00 \mathrm{~m} / \mathrm{sec}$ \\
\hline
\end{tabular}

pedestrians. The best sinks to handle events are always selected using the shortest path [14] strategy. Table I summarizes the values of the parameters used in the simulation study.

\section{B. Baselines and performance metrics}

For positioning of the mobile sinks, four different strategies are compared. In addition to our approaches PcP and PmP, the Weighted Positioning (WP) and Random Positioning (RP) approaches are simulated with the scenarios in which communication constraint does (w/ CC) or does not (w/o CC) exist for the mobile sinks. In case of no communication constraint, we assume that a sink will be handling the event without any collaboration with others. This assumption relaxes the problem because the sinks are assumed to have knowledge about an event and the positions of each other without direct data transmission. Therefore, the strategies certainly produce better outcomes for w/o CC case. Knowing that forming a connected topology is necessary, we still carried out simulations for w/o $\mathrm{CC}$ case to observe how communication constraint affects placement and coverage performance of mobile sinks.

In WP approach, the sinks are placed at the attractions with higher event probabilities according to the vertex weight values $w\left(v_{i}\right)$ of graph $G$. Since the mobile sinks are connected, we again use $S\left[G^{c}\right]$ for the candidate sink positions. Among the candidates, the one which has the vertices with higher weights is chosen for sink placement. The sum of the weights are computed for the vertices of a connected subgraph and the one with the highest sum is marked as the best candidate. In the random positioning (RP) approach, the locations for mobile sinks are selected randomly.

Throughout the simulations, we considered two performance metrics: 1) the average event handling time defined as the travel time for the selected mobile sinks to reach the events on average and 2) the success ratio, the ratio of the times the sink reaches the event before the active time of the event expires.

\section{Performance results}

1) Sink positioning strategies: We analyzed event handling times and success ratios of PcP, PmP, WP and RP under biased and random event distribution models. While the events occur at the attractions according to the event probabilities in the biased event distribution model [14], random model assumes them to be occurring anywhere evenly. Total of five sinks are placed in the theme park and the results of the experiments are extracted from 100 simulation runs for each setting.

The performance results for the event handling time using biased model are shown in Fig. 4-a. The results reveal that PcP and PmP are the winners while WP is slightly better than RP. This can be attributed to the fact that the events are generated based on weights and WP approach is the most suitable for such cases. Comparing our approaches, since we consider the average event handling time as opposed to maximum time as our metric, PmP performs better than PcP. In particular, since the events are not evenly distributed, minimizing the total distance metric in PmP would lead to reduced average event handling times. The RP strategy is the worst in all cases since it does not take the weights of the attractions or the condition of the roads into account.

The simulations are also conducted for evaluating the performance of the approaches for the random event distribution case. The results shown in Fig. 4-b indicate that the best two approaches for random event distribution model are PcP and PmP. Compared to the biased case, we see that the gap between $\mathrm{PcP}$ and $\mathrm{PmP}$ is almost negligible. This is due to the fact that random events occur at any attraction independent from their weights and thus PmP's placement based on the weights does not apply here. PcP, on the other hand, places sinks in such a way that none of them stay very far away from any attractions. Due to even distribution of events at attractions, $\mathrm{PcP}$ and PmP perform similar. Another observation about the results is that the order of RP and WP changes. WP has slightly worse performance as opposed to RP due to its limitation for sink placement. In WP, mobile sinks always placed in attractions with higher weight values whereas in RP the sinks can be placed on any subgraph which brings flexibility. In addition, the performance gap between WP and the proposed approaches increases compared to the previous results (Fig. 4a). This shows that our approaches are much suitable for events occurring by the random distribution.

We observed that in all cases lack of communication constraint boosts the performance significantly and reduces the event handling times. This is due to the availability of more locations for the placement of sinks. Nonetheless, these experiments were performed for five sinks and with the increased number of sinks the gap could be diminished. We will perform separate experiments for such cases next.

Looking at the success ratio performance, we observe that the situation is more critical for random cases. In the biased case, the success ratios for our approaches are slightly better since in most cases the sinks were able to reach the incident locations before the active time expires (e.g., even in the middle of the event). However, for the random case, the moving time to incidents has big gaps and these gaps also affect the success ratio and causes WP and RP to miss the events.

2) Effects of the number of mobile sinks: In the second set of experiments, we evaluated the effect of the number of mobile sinks on the event coverage performance of $\mathrm{PcP}$ and PmP. The experiments are conducted with mobile sinks ranging from 1 to 14 . The random event distribution model is used to generate the events on top of the attractions.

As it can be observed in Fig. 4-c, the success ratio of 
a) Biased event distribution with 5 sinks
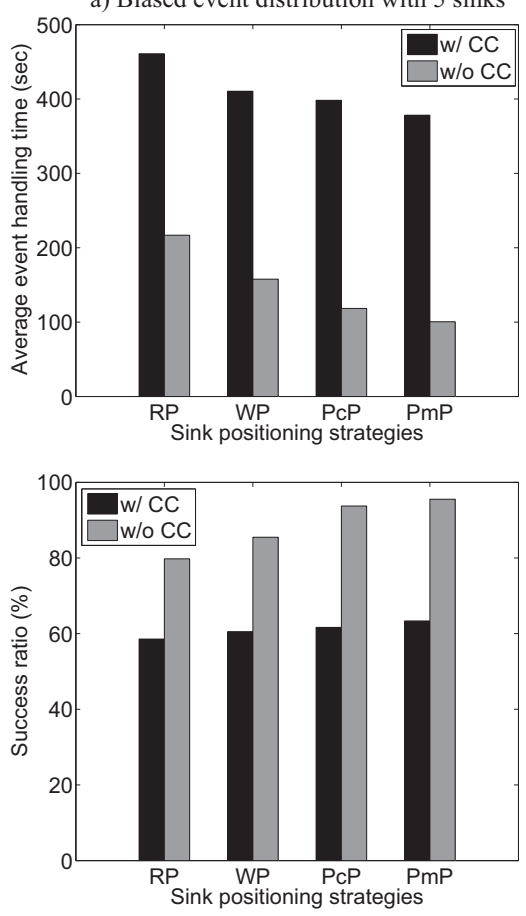

b) Random event distribution with 5 sinks
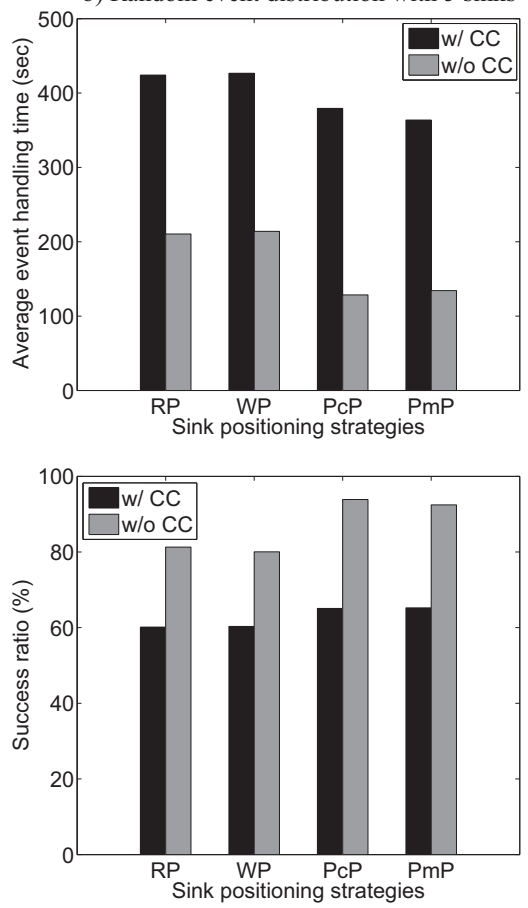

c) 1 to 14 sinks, random event distribution
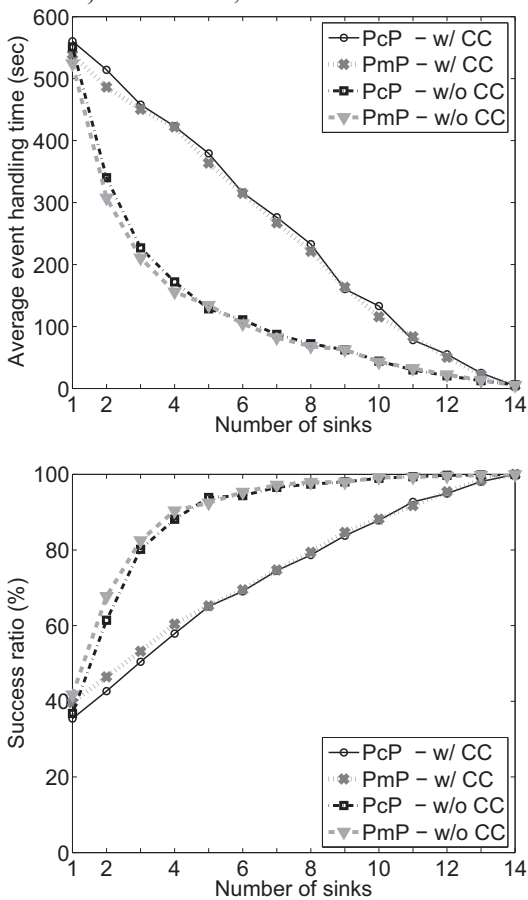

Fig. 4. Average event handling times and success ratios for Random Positioning (RP), Weighted Positioning (WP), p-center positioning (PcP), and p-median positioning (PmP). Comparisons with a) biased event distribution b) random event distribution and c) various number of mobile sinks.

$\mathrm{PcP}$ and PmP strategies are getting higher with the increasing number of mobile sinks. While the performance of PmP is slightly better than PcP for small numbers of sinks, the gap closes as the number of mobile sinks increases. With the increased number of sinks, both approaches can identify sinks within the similar distances from the events and thus the event handling times do not change significantly for PcP and PmP. Again, under the unconstrained cases, PcP w/o CC and PmP w/o CC provide better results since they assume no need for a connected topology with the sinks. However, as the network becomes saturated with more sinks, the gap between the unconstrained and constrained cases is diminished. Overall, the results of the experiments suggest that either PcP or PmP can be used if a certain number of mobile sinks are already available.

\section{CONCLUSION}

In this paper, we focused on the challenge of the event coverage in theme parks. We modeled the attractions and the roads in the theme park with a dynamic weighted graph model. We applied a $p$-center approach to solve the problem on top of this model. We proposed a new variant, communicationconstrained p-center problem, and an exact algorithm to solve it. Based on the algorithm, we positioned the mobile sinks and update their positions in the theme park using the proposed $\mathrm{p}$-center positioning $(\mathrm{PcP})$ and $\mathrm{p}$-median positioning $(\mathrm{PmP})$ approaches. The evaluation of the approaches with respect to two other baselines indicated that the event handling times of the mobile sinks reduce while success ratios significantly increase.

As a future work, we are planning to develop a heuristic algorithm with reduced complexity considering theme parks with large number of attractions.

\section{REFERENCES}

[1] O. Kariv and S. L. Hakimi, "An algorithmic approach to network location problems. i: The p-centers," SIAM Journal on Applied Mathematics, vol. 37, no. 3, pp. 513-538, 1979.

[2] M. Younis and K. Akkaya, "Strategies and techniques for node placement in wireless sensor networks: a survey," Ad Hoc Networks, vol. 6, no. 4, pp. 621-655, June 2008 .

[3] O. Kariv and S. L. Hakimi, "An algorithmic approach to network location problems. i: The p-medians," SIAM Journal on Applied Mathematics, vol. 37, no. 3, pp. 539-560, 1979.

[4] F. Aykut Özsoy and M. Ç. Pınar, "An exact algorithm for the capacitated vertex p-center problem," Computers \& Operations Research, vol. 33, no. 5, pp. 1420-1436, December 2006.

[5] J. Reese, "Solution methods for the p-median problem: An annotated bibliography," Networks, vol. 48, no. 3, pp. 125-142, October 2006.

[6] W. C.-K. Yen, "The connected p-center problem on block graphs with forbidden vertices," Theoretical Computer Science, vol. 426, pp. 13-24, April 2012.

[7] W. C.-K. Yen and C.-T. Chen, "The connected p-center problem with extension," in Proc. of the JCIS'06, October 2006.

[8] W. C.-K. Yen and C.-T. Chen, "The $p$-center problem with connectivity constraint." Applied Mathematical Sciences, vol. 1, no. 27, pp. 13111324, 2007.

[9] D. Zhou and J. Gao, "Maintaining approximate minimum steiner tree and k-center for mobile agents in a sensor network," in Proc. of the IEEE INFOCOM'10, March 2010, pp. 511-515.

[10] K. Akkaya and M. F. Younis, "COLA: A coverage and latency aware actor placement for wireless sensor and actor networks," in VTC Fall, February 2006, pp. 1-5.

[11] A. Boukerche, B. Turgut, N. Aydin, M. Ahmad, L. Bölöni, and D. Turgut, "Routing protocols in ad hoc networks: a survey," Computer Networks (Elsevier), vol. 55, no. 13, pp. 3032-3080, September 2011.

[12] D. Avis and K. Fukuda, "Reverse search for enumeration," Discrete Applied Mathematics, vol. 65, no. 13, pp. 21 - 46, 1996.

[13] G. Solmaz and D. Turgut, "Event coverage in theme parks using wireless sensor networks with mobile sinks," in Proc. of the IEEE ICC'13, June 2013, pp. 115-119.

[14] G. Solmaz and D. Turgut, "Optimizing event coverage in theme parks," Wireless Networks (WINET) Journal, vol. 20, no. 6, pp. 1445-1459, August 2014. 